\title{
Proof of Concept: Design and Initial Evaluation of a Device to Measure Gastrointestinal Transit Time
}

\author{
Robert H. Wagner ${ }^{1}$, Bital Savir-Baruch ${ }^{1}$, James R. Halama ${ }^{1}$, Mukund Venu ${ }^{2}$, Medhat S. Gabriel ${ }^{1}$, and Davide Bova ${ }^{1}$ \\ ${ }^{1}$ Section of Nuclear Medicine, Department of Radiology, Loyola University Medical Center, Maywood, Illinois; and ${ }^{2}$ Section of \\ Gastroenterology, Department of Internal Medicine, Loyola University Medical Center, Maywood, Illinois
}

\begin{abstract}
Chronic constipation and gastrointestinal motility disorders constitute a large part of a gastroenterology practice and have a significant impact on a patient's quality of life and lifestyle. In most cases, medications are prescribed to alleviate symptoms without there being an objective measurement of response. Commonly used investigations of gastrointestinal transit times are currently limited to radiopaque markers or electronic capsules. Repeated use of these techniques is limited because of the radiation exposure and the significant cost of the devices. We present the proof of concept for a new device to measure gastrointestinal transit time using commonly available and inexpensive materials with only a small amount of radiotracer. Methods: We assembled gelatin capsules containing a ${ }^{67} \mathrm{Ga}-$ citrate-radiolabeled grain of rice embedded in paraffin for use as a point-source transit device. It was tested for stability in vitro and subsequently was given orally to 4 healthy volunteers and 10 patients with constipation or diarrhea. Imaging was performed at regular intervals until the device was excreted. Results: The device remained intact and visible as a point source in all subjects until excretion. When used along with a diary of bowel movement times and dates, the device could determine the total transit time. The device could be visualized either alone or in combination with a barium small-bowel follow-through study or a gastric emptying study. Conclusion: The use of a point-source transit device for the determination of gastrointestinal transit time is a feasible alternative to other methods. The device is inexpensive and easy to assemble, requires only a small amount of radiotracer, and remains inert throughout the gastrointestinal tract, allowing for accurate determination of gastrointestinal transit time. Further investigation of the device is required to establish optimum imaging parameters and reference values. Measurements of gastrointestinal transit time may be useful in managing patients with dysmotility and in selecting the appropriate pharmaceutical treatment.
\end{abstract}

Key Words: whole gut transit scintigraphy; gastrointestinal scintigraphy; small-bowel transit; colon transit; constipation

J Nucl Med Technol 2017; 45:230-235

DOI: $10.2967 /$ jnmt.117.192377

Received Feb. 23, 2017; revision accepted Jun. 13, 2017.

For correspondence or reprints contact: Robert H. Wagner, Loyola University Medical Center, 2160 S. First Ave., Maywood, IL 60153.

E-mail: rwagner@lumc.edu

Published online Jul. 13, 2017.

COPYRIGHT (c) 2017 by the Society of Nuclear Medicine and Molecular Imaging.
$\mathbf{T}$ he investigation of gastrointestinal function and motility can be traced back to the very early days of radiology. Shortly after the discovery of x-rays by Roentgen in 1895 , stomach motility was explored by Lindeman (1) and the physiology of swallowing and peristalsis was described by Cannon (2,3). Bismuth, which was used by Rieder in 1904 as a contrast medium to explore the stomach and bowel motility, was replaced in 1910 by the less toxic barium $(4,5)$. For decades, a variety of barium studies became the standard technique to investigate the anatomy and physiology of the gastrointestinal system.

Some previously described methods to determine gastrointestinal transit time included having the patient swallow glass beads ( 6 ), dyes (7), or other nondigestible materials (8) and noting the time that they first appeared in the stool. Radiopaque markers have also been used successfully but require serial radiographs and the associated repeated radiation exposure (9). More recently, a wireless capsule was used to measure transit time (10). This technique is expensive, however, and the large capsule is sometimes difficult to swallow.

Early scintigraphic techniques to evaluate gastric emptying used ${ }^{51} \mathrm{Cr}(11)$ or either ${ }^{113 \mathrm{~m}} \mathrm{In}$ - or ${ }^{99 \mathrm{~m}} \mathrm{Tc}$-labeled diethylenetriaminepentaacetic acid in saline (12). In 1976, Meyer et al. described the use of ${ }^{99 \mathrm{~m}}$ Tc-labeled chicken liver as a marker of solid-food emptying from the stomach (13). Today, the Society of Nuclear Medicine and Molecular Imaging procedure guideline for adult solid-meal gastric emptying recommends $118 \mathrm{~mL}$ (4 oz) of cooked liquid egg white, 2 slices of toasted white bread, $30 \mathrm{~g}$ of jelly or jam, and $120 \mathrm{~mL}$ of water as the standard meal (14). A technique using ${ }^{111}$ In in activated charcoal in a capsule coated with $\mathrm{pH}$-sensitive methacrylate has also been described to investigate gastrointestinal transit time (15). Once the methacrylate dissolves in the terminal ileum, the contents of the capsule are released and mix with the contents of the large bowel and the geometric center of the activity is determined. A scintigraphic technique using liquid tracer sealed in plastic tubing was described in 2005 (16). More recently, scintigraphic studies have been recognized as an effective method of measuring gastrointestinal motility, and new Current Procedural Terminology codes have been made available. The studies are described in a practice guideline 
issued jointly by the Society of Nuclear Medicine and Molecular Imaging and the European Association of Nuclear Medicine (17). This area of research is evolving, and application of these studies in various diseases was recently described (18).

Patients with motility disorders represent a significant part of a gastroenterologist's practice. Irritable bowel disease has a significant impact on patient lifestyle and quality of life. An estimated 2.5 million visits to the physician annually in the United States are for constipation (19), and over $\$ 800$ million is spent on laxative use (20). That figure is for prescription laxatives and would be even higher if over-the-counter preparations were included.

Dysmotility may be caused by medications, an underlying allergy, or inadequate hydration; may have an organic or psychogenic origin; or may have several origins. The etiologies are often difficult to identify, and a variety of attempts are usually made to correct the symptoms, with little objective evidence of results. In current practice, achieving subjective improvement of symptoms often demands the introduction and modification of therapy. This trial-and-error approach leads to prolonged exposure to multiple different medications, which may not be without consequence. Indeed, even if transit time is normalized, patients may continue to have complaints. The ability to objectively measure gastrointestinal transit using an inexpensive, safe, and easy-to-perform procedure with a low radiation burden would benefit this patient group. An objective measurement could help identify the effect of the interventional medication, document normalization of transit, and perhaps direct the physician to other causes of the symptoms.

Our goal was to develop a point-source transit device for the measurement of gastrointestinal transit time. With the exception of the isotope, the device would ideally be constructed using inexpensive and commonly available materials.

\section{MATERIALS AND METHODS}

\section{Device Design}

When designing a device to follow the movement of solids as they pass through the gastrointestinal tract, we followed the principles of radiopharmacy (21). The device needed to reflect the underlying physiology without changing it. The device also had to be biologically inert, nontoxic, nonabsorbable, and readily moved with the gastrointestinal contents. It needed to have no appreciable bulk and be easily detected and measurable for many days in patients with slower gastrointestinal motility.

Our first consideration was to select the ideal isotope. The readily available isotopes that have longer half-lives and are amenable to $\gamma$-camera imaging are ${ }^{201} \mathrm{Tl},{ }^{67} \mathrm{Ga}$, and ${ }^{111} \mathrm{In}$. ${ }^{201} \mathrm{Tl}$ was discounted because of its lower energy. If the device were ever to be used in combination with a ${ }^{99 \mathrm{~m}} \mathrm{Tc}$ gastric emptying study, the ${ }^{201} \mathrm{Tl}$ activity might be difficult to detect. Both ${ }^{111}$ In and ${ }^{67} \mathrm{Ga}$ would be effective isotopes for long-term imaging and could easily be distinguished from background ${ }^{99 \mathrm{~m}} \mathrm{Tc}$ activity. We chose ${ }^{67} \mathrm{Ga}$-citrate for this investigation primarily because it cost less than ${ }^{111}$ In-chloride.
The next consideration was to identify the vehicle to carry the isotope through the gastrointestinal tract. A nondigestible material was necessary. Most plastics are biologically inert, but empty plastic capsules to contain the tracer were not easily found. Paraffin is a nonreactive material that is impervious to both acid and alkali. On further investigation, we also found that paraffin remains solid at physiologic temperatures, with a melting point of approximately $58^{\circ} \mathrm{C}-62^{\circ} \mathrm{C}(22)$. An additional benefit is that paraffin is an established nontoxic material that is Food and Drug Administrationapproved and widely used in food preparation and storage (23). Commonly available and inexpensive, it is an ideal material that would remain unaltered as it passes through the gastrointestinal tract. Unfortunately, the isotope and paraffin are immiscible and could not be easily combined to form a single source. A third component would be needed to contain the isotope within the paraffin.

The majority of any radiopharmaceutical as dispensed from a radiopharmacy is water, with relatively few atoms of radioactive material. Evaporation of the liquid phase from the radiopharmaceutical would leave the radioactive atoms behind, but these would be impossible to manipulate. A porous or hygroscopic material could absorb the radiotracer and allow for easier manipulation. Although a variety of porous materials were considered, we continued to pursue the use of materials that are commonly available and nontoxic. Grains of food-grade rice are hygroscopic, small, and easy to manipulate. We investigated and developed a technique to evaporate the liquid phase of the radiopharmaceutical and concentrate the activity within a grain of rice.

Simply dipping the grain of rice in melted paraffin was insufficient to create a uniform coating and might not protect the grain sufficiently to prevent digestion as it passes through the gastrointestinal tract. A method to easily and uniformly contain the grain within the paraffin was required. We chose to use a standard gelatin capsule as a container. After filling the gelatin capsule with melted paraffin, the grain could be placed into the center and more liquid paraffin added to completely encase the grain. With this method, at least 1-2 mm of paraffin would surround the grain (Fig. 1). We felt this amount to be sufficient for protection and proceeded with testing of the integrity of the device.

\section{In Vitro Stability Testing}

In the earliest stability testing of the device, the grain of rice was stained with blue food dye to check for leakage. During this testing, we found that the blue dye made the grain easier to see and manipulate when it was being inserted into the melted paraffin. We therefore continued using the blue dye when constructing devices for further stability tests and for the clinical evaluation.

Subsequently, in vitro stability was tested on 5 copies of the device containing various levels of ${ }^{99 \mathrm{~m}} \mathrm{Tc}$-pertechnetate activity ranging from 17.2 to $22.6 \mathrm{MBq}(464-610 \mu \mathrm{Ci})$ (average, $18.8 \mathrm{MBq}$ [509 $\mu \mathrm{Ci}]$ ). These 5 devices were placed in a beaker containing $1 \mathrm{~L}$ of water and warmed to $45^{\circ} \mathrm{C}\left(113^{\circ} \mathrm{F}\right)$ for $3 \mathrm{~h}$ with continuous stirring by a magnetic rod. A 3-mL sample of the water was obtained before testing and after 1,2, and $3 \mathrm{~h}$ of testing and measured in a well counter for 3 min to check for leakage.

In a standard ${ }^{67} \mathrm{Ga}$-citrate study using a $185-\mathrm{MBq}$ intravenous dose $(5 \mathrm{mCi})$, approximately $10 \%(18.5 \mathrm{MBq})$ of the dose is excreted through the gastrointestinal tract (24). Since the device uses approximately $20 \%$ of the above activity expected in the gastrointestinal tract, dose estimates for the stomach, small intestine, 


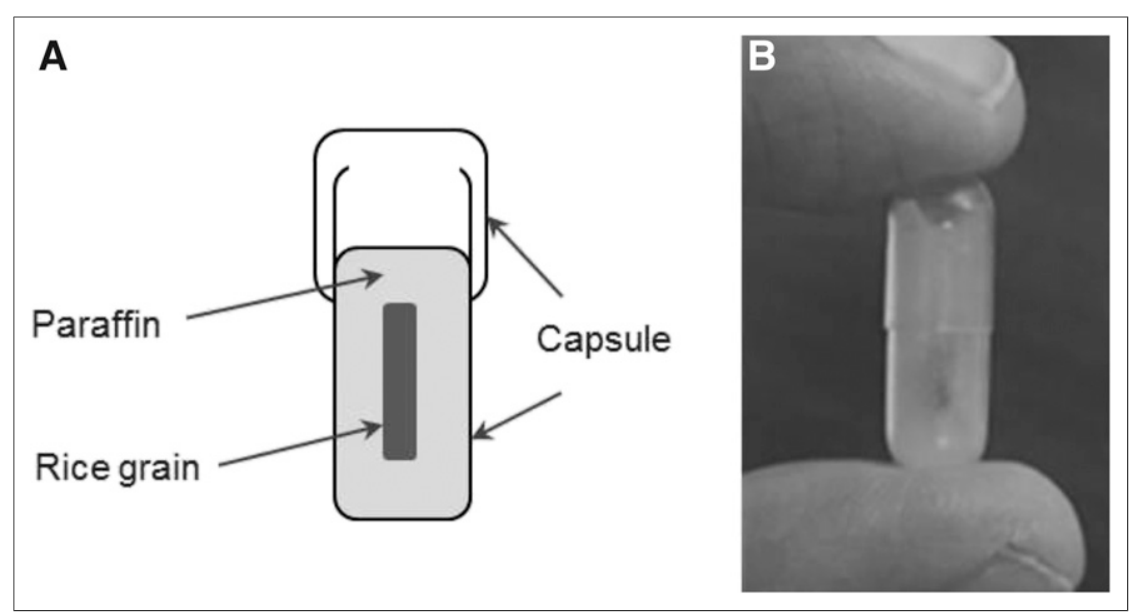

FIGURE 1. (A) Device design includes gelatin capsule containing central core of paraffin-surrounded radiolabeled rice grain. (B) Final assembled device is small and easily swallowed.

upper large intestine, and lower large intestine are 2, 3.6, 5.6, and $9 \mathrm{mGy}$, respectively.

In the unlikely event that the device lodges in the appendix or a diverticulum and delivers the entire dose of radiation at that location, we estimated the dose at 0.97 Gy. Such a dose is significantly less than a single fraction of radiotherapy and would be delivered over a much longer period. Even in this worst-case scenario, the dose would be unlikely to cause an acute local reaction in the appendix or diverticulum.

\section{Clinical Evaluation}

Population. The study population consisted of 4 healthy volunteers and 10 patients with gastrointestinal complaints. All 14 subjects were older than $18 \mathrm{y}$. The volunteers had no gastrointestinal complaints. The patients had been referred by a gastroenterologist for either a nuclear medicine gastric emptying study or a barium small-bowel follow-through study. Their gastrointestinal complaints were either constipation (9 patients) or diarrhea (1 patient). None of the subjects had undergone any prior gastrointestinal surgery other than appendectomy or cholecystectomy. All fasted from midnight until the study. The patients stopped taking any gastrointestinal motility-related medications $24 \mathrm{~h}$ before the study. The institutional review board of Loyola University Medical Center approved the human investigations, and all subjects signed an informed consent form. The radiation control committee of Loyola University Medical Center approved the investigational use of the device.

Dose. The first of the volunteers received a device containing $6.62 \mathrm{MBq}(179 \mu \mathrm{Ci})$ of ${ }^{67} \mathrm{Ga}$-citrate. On the basis of the intensity seen on the images, the activity in all subsequent devices was decreased to $2.39-3.55 \mathrm{MBq}(64.7-96.0 \mu \mathrm{Ci})$.

Imaging. Two-minute anterior projection images were obtained using a Forte $\gamma$-camera (Philips) with a medium-energy collimator. All subjects underwent imaging as soon as they had swallowed the device; again at 15, 30, 45, and 60 min afterward; and then every hour for $6 \mathrm{~h}$, when they resumed their normal diet and (for the patients) medications. They were asked to return for imaging on each subsequent day until the device was no longer visible on images, indicating it had been excreted.
Three of the patients swallowed the device with $500 \mathrm{~mL}$ of barium and underwent a concurrent barium small-bowel follow-through study. The remaining 7 patients swallowed the device while eating the standard radiolabeled meal and underwent a concurrent nuclear medicine gastric emptying study with a dual-isotope ${ }^{99} \mathrm{~m} \mathrm{Tc}$ and ${ }^{67} \mathrm{Ga}$ ) acquisition. The volunteers swallowed the device with $237 \mathrm{~mL}$ ( $8 \mathrm{oz}$ ) of water and did not undergo any concurrent imaging.

Knowing the location of the device is important if small-bowel transit time is to be determined. The volunteers and the 3 patients who had a concurrent barium study were imaged with a ${ }^{57} \mathrm{Co}$ sheet source behind them to help identify the anatomic location of the device. The sheet source was not used for the 7 patients who had a concurrent gastric emptying study; instead, digital fusion (Hermes Medical Solutions) of the planar images acquired using the dual-isotope ${ }^{99 \mathrm{~m}} \mathrm{Tc}$ and ${ }^{67} \mathrm{Ga}$ windows was used to help locate the device.

Stability. The device was considered stable if the final image displaying activity before excretion of the device showed a point source. If the integrity of the device failed, the leaked activity would presumably be seen as a "blush" and be dispersed within the bowel contents.

Total Gastrointestinal Transit Time. All subjects kept a diary of the dates and times of their bowel movements. The device excretion time was defined as the time of the first bowel movement after the last imaging session showing the device. Total gastrointestinal transit time was calculated as the difference between the time the device was swallowed and the time it was excreted. The device was not recovered as part of this study.

\section{RESULTS}

\section{Stability}

The device was found to be stable when warmed in the water bath. None of the water-bath samples had activity above background levels. Although the gelatin capsule dissolved, the central paraffin-rice combination remained intact. Approval of the device by the institutional review board and the radiation control committee was based on these initial stability findings and the nonsignificant risk associated with its use. We report here on its stability and detectability only regarding measurement of gastrointestinal transit times.

In all 14 subjects, the device remained visible, without apparent leakage, until the subject either withdrew from the study or the imaging was completed.

\section{Total Gastrointestinal Transit Time}

All 4 volunteers excreted the device by $24 \mathrm{~h}$, with the total gastrointestinal transit time averaging $15 \mathrm{~h} 57 \mathrm{~min}$ (SD, $7 \mathrm{~h} 18 \mathrm{~min}$; range, $7 \mathrm{~h} 0 \mathrm{~min}$ to $22 \mathrm{~h} 18 \mathrm{~min}$ ). One volunteer excreted the device at $7 \mathrm{~h}$ and on questioning was found to have consumed several large cups of coffee after taking the device. Despite the hot coffee, the device remained intact and visible on images at 3 and $5 \mathrm{~h}$. 
One of the patients with constipation, who underwent a concurrent barium small-bowel follow-through study, refused further imaging after $4 \mathrm{~h}$. Another patient with constipation, who underwent a concurrent nuclear medicine gastric emptying study, refused further imaging after $24 \mathrm{~h}$. The remaining 8 patients continued with imaging until the device had been excreted. Total gastrointestinal transit time for the 7 patients with constipation averaged $40 \mathrm{~h} 45 \mathrm{~min}$ (SD, $18 \mathrm{~h} 13 \mathrm{~min}$; range, $9 \mathrm{~h} 50 \mathrm{~min}$ to $67 \mathrm{~h} 43 \mathrm{~min}$ ). The transit times for all subjects are summarized in Table 1.

Although the complaint for patient 9 was constipation, this patient had 3 bowel movements between the imaging session at $6 \mathrm{~h}$ and the imaging session the next day, which no longer showed the device. Excretion may have occurred at the time of any of these 3 bowel movements. In accord with our definition, we based the total gastrointestinal transit time on the time of the first bowel movement after the imaging session at $6 \mathrm{~h}$; that is, the bowel movement occurring at $9 \mathrm{~h} 50 \mathrm{~min}$ after administration. The 2 subsequent bowel movements occurred at $22 \mathrm{~h}$ and at $24 \mathrm{~h}$ $30 \mathrm{~min}$ after administration. It is possible that excretion occurred at one of these time points instead.

\section{Location Determination}

Use of a sheet source behind the subject allowed for general localization of the device to the upper abdomen, mid abdomen, lateral abdomen, or pelvis but did not with confidence identify the time that the device entered the ascending colon (Fig. 2). Digital fusion of the planar images acquired using the dual-isotope ${ }^{99 \mathrm{~m}} \mathrm{Tc}$ and ${ }^{67} \mathrm{Ga}$ win-

TABLE 1

Total Gastrointestinal Transit Time for Each Subject

\begin{tabular}{|c|c|c|c|}
\hline Subject no. & Symptoms & Transit time & $\begin{array}{l}\text { Gastric } \\
\text { emptying } \\
\text { result }\end{array}$ \\
\hline \multicolumn{4}{|l|}{ Volunteers } \\
\hline 1 & None & $21 \mathrm{~h} 30 \mathrm{~min}$ & \\
\hline 2 & None & $22 \mathrm{~h} 18 \mathrm{~min}$ & \\
\hline 3 & None & $13 \mathrm{~h} 0 \mathrm{~min}$ & \\
\hline 4 & None & $7 \mathrm{~h} 0 \mathrm{~min}$ & \\
\hline Average & & 15 h $57 \mathrm{~min}^{*}$ & \\
\hline \multicolumn{4}{|l|}{ Patients } \\
\hline 1 & Constipation & 43 h $33 \mathrm{~min}$ & \\
\hline 2 & Constipation & Withdrew & \\
\hline 3 & Constipation & 46 h 46 min & \\
\hline 4 & Constipation & $21 \mathrm{~h} 39 \mathrm{~min}$ & Normal \\
\hline 5 & Diarrhea & 21 h 39 min & Normal \\
\hline 6 & Constipation & 67 h $43 \mathrm{~min}$ & Normal \\
\hline 7 & Constipation & $46 \mathrm{~h} 21 \mathrm{~min}$ & Normal \\
\hline 8 & Constipation & $26 \mathrm{~h} 2 \mathrm{~min}$ & Accelerated \\
\hline 9 & Constipation & $9 \mathrm{~h} 50 \mathrm{~min}$ & Delayed \\
\hline 10 & Constipation & Withdrew & Normal \\
\hline \multicolumn{2}{|l|}{ Average } & \multicolumn{2}{|l|}{$40 \mathrm{~h} 45 \min ^{\dagger}$} \\
\hline \multicolumn{4}{|c|}{$\begin{array}{l}{ }^{*} \text { May be artificially low since volunteer } 4 \text { drank several cups of } \\
\text { coffee after swallowing device. } \\
{ }^{\dagger} \text { May be artificially low since patient } 9 \text { had } 3 \text { bowel movements } \\
\text { after last imaging session showing device. }\end{array}$} \\
\hline
\end{tabular}

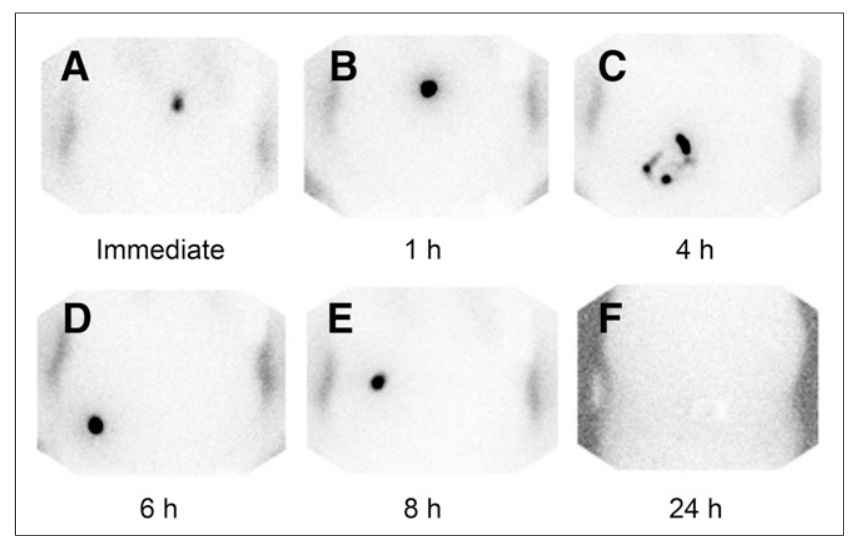

FIGURE 2. Images of device obtained using ${ }^{57} \mathrm{Co}$ sheet source behind subject. (A) Device is seen immediately after being swallowed. (B) At $1 \mathrm{~h}$, when device is used alone it is difficult to be confident that it has entered small bowel. (C) At $4 \mathrm{~h}$, motion of device within small bowel during image acquisition can be seen. (D) At $6 \mathrm{~h}$, device is likely at ileocecal junction. (E) At $8 \mathrm{~h}$, device is likely in ascending colon. (F) At $24 \mathrm{~h}$, device has been excreted.

dows allowed greater confidence about whether the device was within the stomach, the small bowel, or the large bowel (Fig. 3).

\section{DISCUSSION}

The currently available nonscintigraphic techniques to measure gastrointestinal transit times either are expensive

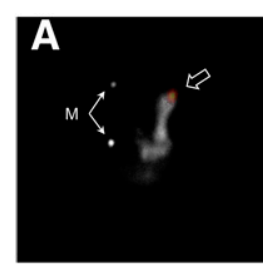

$1 \mathrm{~h}$

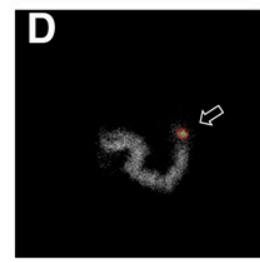

$24 \mathrm{~h}$

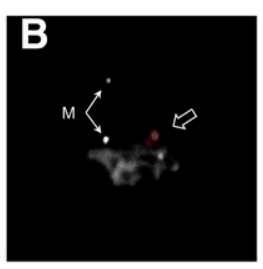

$4 \mathrm{~h}$

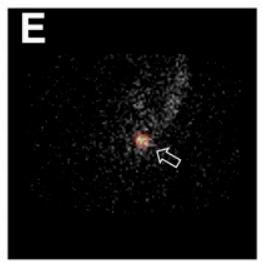

$48 \mathrm{~h}$

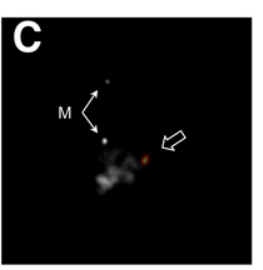

$6 \mathrm{~h}$

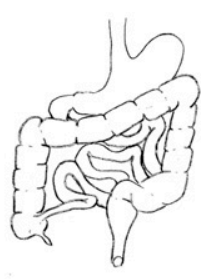

FIGURE 3. Dual-isotope acquisitions with digitally fused images (as well as diagram of normal anatomy to help identify device location) in patient with history of constipation. Use of dual-isotope acquisitions in conjunction with gastric emptying study allows location of device to be defined more clearly. Device (open arrows) can be seen $1 \mathrm{~h}$ after being swallowed $(\mathrm{A})$, is still in stomach at $4 \mathrm{~h}(\mathrm{~B})$, is visible in small bowel at $6 \mathrm{~h}$ $(\mathrm{C})$, is in transverse colon at $24 \mathrm{~h}(\mathrm{D})$, and is in rectosigmoid region at $48 \mathrm{~h}(\mathrm{E})$. An image obtained at $72 \mathrm{~h}$ showed no activity. $\mathrm{M}=$ point source markers placed on patient to right of midline approximately at levels of nipple and umbilicus to ensure consistent patient positioning between images. 
(wireless capsules) or require multiple abdominal radiographs and the associated radiation exposure (radiopaque markers). Because markers using barium or other liquids mix with the gastrointestinal contents, such markers may not reflect the physiologic motility of solids, making transit time calculations difficult. Markers using indigestible foods or dyes have similar limitations.

Our point-source transit device was inexpensive and easy to construct, and it comprises commonly available, nontoxic food-grade materials. It remained visible in vivo for several days, and although the number of subjects was admittedly small, the device proved stable in all. Evaluation of total gastrointestinal transit time was simple, requiring only a few additional imaging time points and a patient diary of bowel movements. To obtain a more complete clinical picture of gastrointestinal motility, the diary might be extended to a week before the beginning of the motility study and a week after its conclusion. In addition, transit times might be better derived if the patients were to discontinue their medications for longer than the $24 \mathrm{~h}$ we required. In patients with diarrhea, our protocol is unlikely to capture the true total gastrointestinal transit time because of the frequency of bowel movements. Further, this frequency would need to be addressed regardless of the transit time. The device is therefore more likely to be beneficial in patients with constipation.

Objective measurements of bowel transit may be useful in evaluating the response to medication since some medications for chronic constipation can be quite expensive. Transit time can be documented at baseline, and any changes due to medication can be used to guide further therapy. Should the transit time normalize after intervention but the symptoms persist, alternatives such as dietary, allergic, or psychogenic etiologies can be considered.

The utility of the device needs to be further explored. Used in combination with a gastric emptying study or other scintigraphic techniques, the device can allow estimation of not just gastric emptying time but total gastrointestinal transit time and, potentially, small-bowel transit time. The protocol, however, need to be optimized to derive these results. At this time, fusion of planar images obtained using dual-isotope imaging allows for the greatest ability to identify the anatomic location of the device.

The timing of the imaging also needs to be optimized. The current continuous $6 \mathrm{~h}$ spent in the department can be demanding for the patient. When the device is used in conjunction with a gastric emptying study, the standard imaging schedule-every $15 \mathrm{~min}$ for the first hour and every hour thereafter for $4 \mathrm{~h}$-would normally be used. If the device is not seen at the ileocecal junction, additional imaging could be performed at 6 or $8 \mathrm{~h}$. The scheduling of delayed imaging at $24 \mathrm{~h}, 48 \mathrm{~h}$, or even later can be more flexible for the patient since the image is used for only correlation with bowel movements to estimate the time of excretion.
A larger study of healthy volunteers is required to more firmly establish the reference range for the device; we therefore continue to accrue patients into our ongoing study. Establishing a reference range would also be helpful in reassuring patients whose transit time falls within this range and in allowing their physicians to pursue other causes for their symptoms.

The point-source transit device that we describe here is considered by the Food and Drug Administration to be a device rather than a radiopharmaceutical (Division of Industry and Consumer Education, Food and Drug Administration; oral communication; January 2014), and the nature of the device is such that it falls into the nonsignificant-risk class (25). However, since no similar device is currently available on the market, near-term future use and clinical investigations will still require institutional review board approval.

\section{CONCLUSION}

We have proved the concept of a new point-source transit device for the measurement of gastrointestinal transit time. Inexpensive and easy to produce from commonly available materials, and using only a small amount of tracer, the device has proved to be stable in vivo and effective in estimating total gastrointestinal transit times for solids. Use of such a device in combination with other scintigraphic techniques may prove valuable. Additional investigation is required to establish the optimum dose, imaging times, and techniques, as well as reference and pathologic values, and a larger study is needed to determine whether the device will be helpful in selecting the proper pharmaceutical therapy for individuals with chronic constipation.

\section{DISCLOSURE}

A portion of this study was supported by a grant from Trinity Healthcare. A U.S. patent application for the device has been submitted (publication date, June 30, 2016; publication number, US20160184466 A1; inventor, Robert Hans Wagner; assignee, Loyola University of Chicago). No other potential conflict of interest relevant to this article was reported.

\section{REFERENCES}

1. Lindemann E. Demonstration of roentgen pictures of the normal and distended stomach. Dtsch Med Wochenschr. 1897;23:266-267.

2. Cannon WB, Moser A. The movements of the food in the œsophagus. Am J Physiol. 1898;1:435-444.

3. Cannon WB. The movements of the stomach studied by means of the roentgen rays. Am J Physiol. 1898;1:358-382.

4. Eisenberg RL, Margulis A. Brief history of gastrointestinal radiology. Radiographics. 1991;11:121-132.

5. Carman RD, Miller A. The roentgenologic determination of gastric motility: with a comparison of the results obtained in a series of cases examined both by the roentgen ray and the test-meal. Arch Intern Med (Chic). 1915;XVI:406428.

6. Alvarez WC, Freedlander BL. The rate of progress of food residues through the bowel. JAMA. 1924;83:576-580. 
7. Dimson SB. Carmine as an index of transit time in children with simple constipation. Arch Dis Child. 1970;45:232-235.

8. Hoelzel F. The rate of passage of inert materials through the digestive tract. Am J Physiol. 1930;92:466-497.

9. Hinton JM, Lennard-Jones JE, Young AC. A new method for studying gut transit times using radiopaque markers. Gut. 1969;10:842-847.

10. Sarosiek I, Selover KH, Katz LA, et al. The assessment of regional gut transit times in healthy controls and patients with gastroparesis using wireless motility technology. Aliment Pharmacol Ther. 2010;31:313-322.

11. Griffith GH, Owen GM, Kirkman S, Shields R. Measurement of rate of gastric emptying using chromium-51. Lancet. 1966;1:1244-1245.

12. Chaudhuri TK. Use of ${ }^{99 \mathrm{~m}} \mathrm{Tc}-\mathrm{DTPA}$ for measuring gastric emptying time. $\mathrm{J} \mathrm{Nucl}$ Med. 1974;15:391-395.

13. Meyer JH, MacGregor IL, Gueller R, Martin P, Cavalieri R. ${ }^{99 m}$ Tc-tagged chicken liver as a marker of solid food in the human stomach. Am J Dig Dis. 1976;21:296-304.

14. Donohoe KJ, Maurer A, Ziessman H, Urbain J, Royal H, Martin-Comin J. Procedure guideline for adult solid-meal gastric-emptying study 3.0. J Nucl Med Technol. 2009;37:196-200.

15. Burton DD, Camilleri M, Mullan BP, Forstrom LA, Hung JC. Colonic transit scintigraphy labeled activated charcoal compared with ion exchange pellets. J Nucl Med. 1997;38:1807-1810.

16. Kekilli E, Yagmur C, Isik B, Aydin OM. Calculating colon transit time with radionuclide-filled capsules in constipated patients: a new method for colon transit study. Abdom Imaging. 2005;30:593-597.
17. Maurer A, Camilleri M, Donohoe K, et al. The SNMMI and EANM practice guideline for small-bowel and colon transit 1.0. J Nucl Med. 2013;54:20042013.

18. Maurer AH. Gastrointestinal motility, part 2: small-bowel and colon transit. J Nucl Med Technol. 2016;44:12-18.

19. Sonnenberg A, Koch TR. Physician visits in the United States for constipation: 1958 to 1986. Dig Dis Sci. 1989;34:606-611.

20. Sanchez MI, Bercik P. Epidemiology and burden of chronic constipation. Can J Gastroenterol. 2011;25(suppl B):11B-15B.

21. Karesh, Stephen M. Principles of radiopharmacy. In: Henkin RE, Bova D, Dillehay GL, et al., eds. Nuclear Medicine. Philadelphia, PA: Mosby; 2006:332349.

22. Material data safety sheet: paraffin wax (granular). Indo Gulf Group website. http://indogulfgroup.com/MSDS/PARAFFIN\%20wax.pdf. Published September 2, 1999. Revised March 18, 2003. Accessed June 29, 2017.

23. \$175.250 paraffin (synthetic). U.S Government Publishing Office website. https:/www.gpo.gov/fdsys/pkg/CFR-2011-title21-vol3/pdf/CFR-2011-title21vol3-sec175-250.pdf. Published April 1, 2011. Accessed June 29, 2017.

24. Gallium citrate Ga 67 injection: Rx only. Mallinckrodt Pharmaceuticals website. www2.mallinckrodt.com/WorkArea/DownloadAsset.aspx?id=470. Revised October 2015. Accessed June 29, 2017.

25. Information sheet guidance for IRBs, clinical investigators, and sponsors: significant risk and nonsignificant risk medical device studies. U.S. Food and Drug Administration website. www.fda.gov/downloads/RegulatoryInformation/ Guidances/UCM126418.pdf. Published January 2006. Accessed June 29, 2017. 